\title{
An Engineering Approach towards Action Refinement
}

\author{
Dick A.C. Quartel ${ }^{\mathrm{a}}$, Luís Ferreira Pires ${ }^{\mathrm{a}}$, Henry M. Franken ${ }^{\mathrm{b}}$, Chris A. Vissers ${ }^{\mathrm{a}, \mathrm{b}}$ \\ (a) Centre for Telematics and Information Technology, University of Twente, \\ PO Box 217, $7500 \mathrm{AE}$ Enschede, the Netherlands \\ (b) Telematics Research Centre, PO Box 217, 7500 AE Enschede, the Netherlands
}

\begin{abstract}
In the abstract modelling of distributed systems we may need methods to replace abstract behaviours by more concrete behaviours which are closer to implementation mechanisms. Furthermore, we may want these methods to preserve the correctness of such a replacement. This paper introduces an approach towards action refinement in which an abstract action is replaced by a concrete activity. This approach is based on a careful consideration of the 'action' and 'causality relation' architectural concepts, which enable an abstract action to be replaced by many alternative concrete activities in a general way. This approach is based on the application of abstraction rules to determine whether a concrete activity conforms to an abstract action, considering the context in which the concrete activity and the abstract action are embedded.
\end{abstract}

\section{Introduction}

During the design process of telematic applications or business processes we may have to replace abstract designs by more concrete designs, in which more detailed design information is defined. We consider the relation between an abstract design and a more concrete design, based on the assumption that a design is a prescription for implementation. An abstract design prescribes what should be implemented, while a more concrete design prescribes how this abstract design should be implemented. The notion of abstract design and the notion of more concrete design are relative notions, since e.g. a more concrete design may be considered as an abstract design in a next design step.

Action refinement is defined as a design operation in which an abstract action is replaced by a concrete activity that conforms to this abstract action. A concrete activity is a composition of multiple actions which are considered to be more concrete than the abstract action.

A conformance relation defines what concrete activities are valid refinements, or implementations, of an abstract action, considering the context in which the concrete activity and the abstract action are embedded. This conformance relation should guarantee that what is prescribed by an abstract behaviour is preserved by a more concrete behaviour, where abstract actions of the abstract behaviour are replaced by more concrete activities in the concrete behaviour.

The precise meaning of a behaviour is defined by the precise meaning of the underlying architectural concepts and their combination rules. The precise meaning of architectural concepts is called architectural semantics [4].

This paper presents an engineering approach towards action refinement that is based on the 'action' and 'causality relation' architectural concepts. The adjective 'engineering' applies to the central role of these architectural concepts in this approach, since they represent the notions that are manipulated by a designer in order to conceive, understand and structure some behaviour. Therefore, the objectives of action refinement and the manipulations which are necessary to perform this design operation are defined in terms of these architectural concepts. This paper presents the conceptual basis to perform action refinement in a general way, although the method is probably not yet complete, in the sense that it is possible that some acceptable refinements are not yet supported by the method.

The remainder of this paper is structured as follows: section 2 recalls the action and causality relation definitions, section 3 defines action refinement and identifies correctness requirements, sections 4 and 5 discuss rules to assess these correctness requirements, section 6 applies these rules to an example, and section 7 presents some conclusions and discusses directions for further research.

\section{Actions and causality relations}

This section briefly presents the basic architectural concepts of action and causality relation. More elaborate discussions can be found in [3] and [2]. 
The action concept. An action models relevant characteristics of some activity in the real world, abstracting from characteristics that are considered irrelevant at the point in the design process where the action is considered. The relevant characteristics are represented by the following action attributes:

- location: the location where the action occurs;

- time: the moment of time when the action occurs;

- information values: the information values that are established in the action;

- retained values: information values established in other actions that happened before the action, and kept by this action for further reference; the union of the information and retained values of an action is called the functionality of this action.

- probability: the probability that the action occurs once it is enabled.

Each action is considered to be an unique occurrence. Therefore, we assume that we can unambiguously refer to an action by using an action identifier.

The concept of causality relation. A causality relation models a causal dependency between action occurrences. The causality relation of an action $a$ defines the causality condition of $a$ in terms of:

- other actions that must have occurred before $a$ : its enabling actions;

- other actions that must not have occurred before or at the same time as $a$ : its disabling actions;

- conditions on the attribute values of the enabling actions;

- constraints on the attribute values of $a$.

Action $a$ is called the result action. Action $a$ is enabled if and only if its causality condition is satisfied. Action $a$ can only occur at the moments of time at which it is enabled. Once action $a$ occurs the attribute values that are established in $a$ can be referred to by other actions enabled by $a$.

Basic causality relations. Two basic causality relations can be identified:

- causality: $b \rightarrow a$, the occurrence of $b$ is a condition for the occurrence of $a$; only if $b$ has occurred $a$ is allowed to occur and can refer to the attribute values of $b$.

- exclusion: $\neg b \rightarrow a$, the non-occurrence of $b$ is a condition for the occurrence of $a$; if $b$ occurs and $a$ has not occurred before it, $b$ disables the occurrence of $a$.

Logical combinations. Logical combinations of enabling and disabling actions can be defined using the logical operators and $(\wedge)$ and $o r(v)$ to represent more complex causality conditions. Two examples are:

- $b \wedge \neg c \rightarrow a$, the occurrence of $b$ and the non-occurrence of $c$ are both conditions for the occurrence of $a$. Action $a$ can refer to the attribute values of $b$;
- $b \vee c \rightarrow a$, either the occurrence of $b$ or the occurrence of $c$ enables the occurrence of $a$. In case both $b$ and $c$ have occurred before $a$ occurs, there is a non-deterministic choice on which of these actions enable $a$. Action $a$ can only refer to the action that enables it, either $b$ or $c$, but not both.

The causality relation of an action does not impose any constraints on the occurrences of its enabling and disabling actions. The causality condition of these actions should be defined separately in their causality relations.

Behaviour definitions. A behaviour is defined by a set of causality relations, one relation per action of the behaviour. Initial actions are enabled from the beginning of the behaviour by the condition denoted as start.

Figure 1 depicts an example behaviour, which consists of four actions $a, b, c$ and $d$. Actions $a, b$ and $\mathrm{c}$ are defined to occur sequentially. In addition, action $a$ enables a timer, called $d$, which may disable the occurrence of $\mathrm{c}$, in case $c$ does not occur before the timer expires.

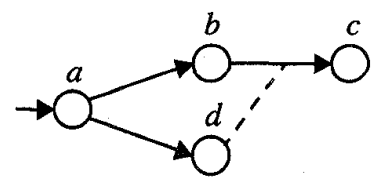

\section{Figure 1: Example behaviour}

The textual representation of the example behaviour of figure 1 is: $B:=\{$ start $\rightarrow a, a \rightarrow b, a \rightarrow d, b \wedge \neg d \rightarrow c\}$. We refrain from explicitly defining action attribute values in this example for the sake of conciseness.

\section{Definition of action refinement}

Action refinement consists of replacing an action of an abstract behaviour by a conforming activity in a concrete behaviour. All actions of an abstract behaviour are called abstract reference actions in the sequel. An activity is a composition of actions that are considered to be more concrete than its corresponding abstract reference action.

Since an abstract reference action can be replaced by many different alternative more concrete activities, and the choice of a specific concrete activity is determined by specific design objectives, this design operation can not be automated in its totality. However one can determine the correctness of this design operation by comparing the abstraction of the concrete activity with the abstract reference action considering their contexts. ${ }^{1}$

Two correctness requirements are identified to determine

\footnotetext{
${ }^{1}$ When comparing behaviours we only consider the following strict equivalence rule: two behaviours are equivalent if they have corresponding actions and corresponding causality relations. Less strict equivalence rules are left for further study.
} 
whether the activity that replaces an abstract reference action is a correct implementation of that action in its context:

1. conformance between an activity and the abstract reference action;

2. proper embedding of an activity in the context of the abstract reference action.

The approach towards requirement (1) is to determine the rules for considering an abstract reference action as an abstraction of an activity, and to apply these rules for assessing whether an activity conforms to an abstract reference action. These rules determine the attribute values which should be assigned to an abstract reference action in order to consider this action as an abstraction of a certain activity, which we call attribute abstraction.

The approach towards requirement (2) is to determine the rules for abstracting from the specific ways an activity relates to other activities and actions, and apply these rules to determine whether specific activities embedded in the concrete behaviour correctly implement the abstract reference action embedded in the abstract behaviour. Requirement (2) is supported by rules for abstracting from the specific embedding of an activity in a concrete behaviour, which we call context abstraction.

Figure 2 depicts the relationships between attribute abstraction, context abstraction and the design choice to be taken in action refinement.

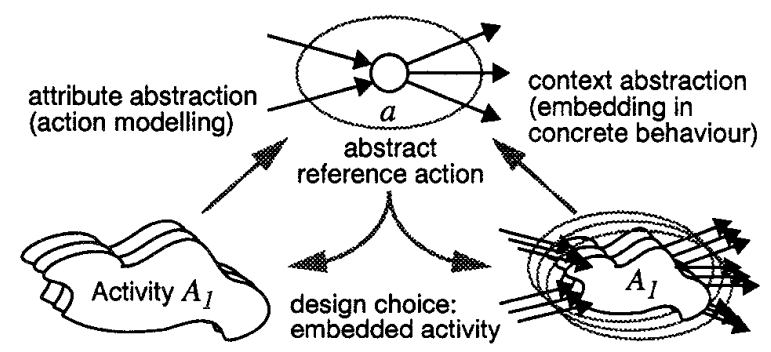

Figure 2: Elements of action refinement

\section{Attribute abstraction}

An action is considered a proper abstraction of an activity if it has attribute values that represent the attributes of the activity, namely the location, value, time and probability of this activity. The conformance between an action and an activity is defined in terms of abstraction rules, which determine the attribute values that an abstract reference action should have in order to be an abstraction of an activity.

These abstraction rules show that the essence of action refinement is the decomposition of at least one of the action attributes of the abstract reference action in multiple action attributes of the actions in the concrete activity. Not only information and retained values, but also the location, time and probability of an abstract reference action may be distributed over actions of an activity. We assume that all attribute values that are possible for an abstract reference action should also be possible in the corresponding concrete activity.

Activities make all their attribute values available through the occurrence of one or more final actions. This follows from the assumption that an activity stops when its essential goals are achieved; i.e. the establishment of all its values. The following generic cases can be distinguished:

- single final action: an activity has a single final action, such that this activity makes all its values available when this final action occurs;

- conjunction of final actions: an activity has multiple independent final actions, such that this activity makes all its values available when all these final actions occur;

- disjunction of final actions: an activity has multiple alternative final actions, such that this activity makes all its values available when one of these final actions occurs;

- any combination of conjunctions and disjunctions of final actions. This case is not explicitly addressed in this paper.

Abstraction rules for the location, time and value attributes are discussed below.

Location. The location of the abstract reference action should be an abstraction of the locations of all actions of the activity, or it should be the same location of all actions of the activity, if they all have the same location. In the former case the location attribute of the abstract action should contain all locations of the actions in the activity, such that these locations are represented as a single more abstract location in the abstract reference action.

Location attribute values can be defined as a hierarchy of locations and sub-locations, similar to e.g. home addresses. Consider the abstract action of delivering mail in a certain town. This action may be distributed over multiple more concrete actions modelling the delivery of mail at different districts of this town. These actions may be further distributed over actions modelling the actual delivery of mail at individual home addresses in these districts. In this way a hierarchy of locations is formed with respect to the location attributes of these actions.

Information values. Considering the three cases of activity ending identified above, the information values of the abstract reference action must be contained in:

- the functionality of the final action, in case of a single final action;

- the union of the functionality of the final actions, in case of a conjunction of final actions;

- the functionality of the actual final action that occurs, in case of a disjunction of final actions. 
This implies that the information values of the abstract reference action are either established or retained in the final action(s) of the concrete activity.

Figure 3 depicts an example of the abstraction of a conjunction of final actions: a parallel interface activity and its corresponding abstract reference action word. The parallel interface activity consists of four independent actions byte ${ }_{1}$, byte $_{2}$, byte $_{3}$ and byte 4 , which establish the octets $o_{1}, o_{2}, o_{3}$ and $o_{4}$, respectively. The information value attribute of word consists of the union of these octets. The location of word is an abstraction of the locations of actions byte ${ }_{i}$.

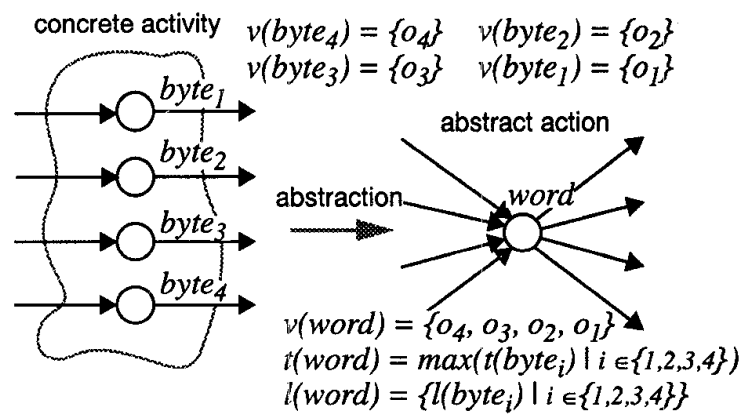

$v($ word $)=$ information value of word $t($ word $)=$ time value of word $r($ word $)=$ retained value of word $\quad$ ( (word $)=$ location value of word

Figure 3: Parallel intterface

Retained values. In analogy to the information value attribute, the retained values of an abstract reference action should be contained in:

- the retained values of the final action, in case of a single final action;

- the union of the retained values of the final actions, in case of a conjunction of final actions;

- the retained values of the actual final action that occurs, in case of a disjunction of final actions.

Figure 4 depicts an example of the abstraction of a serial interface activity with a single final action. The serial interface activity consists of the sequential composition of four actions byte $_{1}$, byte $_{2}$, byte $_{3}$ and byte ${ }_{4}$, which establish the octets $o_{1}, o_{2}, o_{3}$ and $o_{4}$, respectively. The information value attribute of word consists of the union of the information and retained values of action byte 4 . The location of word is the location of actions byte ${ }_{i}$, which are supposed to occur at the same location in this example.

In addition we could consider that the retained value attribute of action word contains the information value of a preceding action word ${ }^{\prime \prime}$, i.e. $r$ (word $)=v\left(\right.$ word $\left.^{\prime}\right)$. In this case, the retained value of word should be also contained in the retained value attributes of byte ${ }_{1}$, byte $_{2}$, byte $_{3}$ and byte $e_{4}$, in order to be used by actions that depend on byte 4 .

Time. The abstract reference action should occur when all values of the activity are available. This implies that the

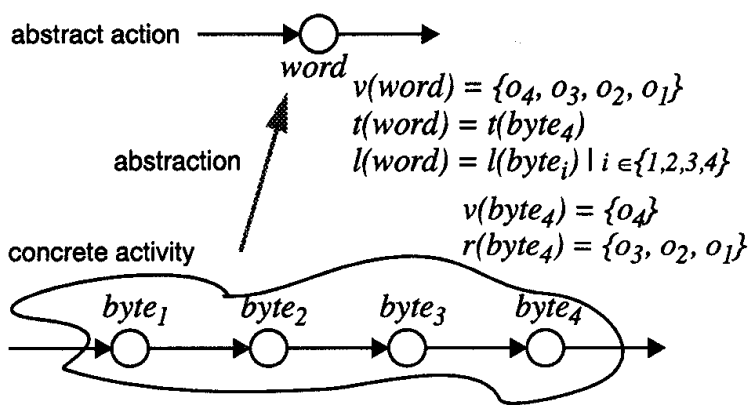

Figure 4: Serial interface

time of the abstract reference action should be:

- the time of the final action, in case of a single final action;

- the time of the latest final action, in case of a conjunction of final actions;

- the time of the actual final action that occurs, in case of a disjunction of final actions.

For example, considering the parallel interface activity of figure 3 , the time attribute of action word is the time of the 'latest' action byte $e_{i}$ that occurs, and in case of the serial interface activity of figure 4 , the time attribute of action word is the time of action byte 4 .

Probability. The probability of an abstract reference action can not be inferred solely from the probability attributes of the final actions, since we also need to consider the actions or activities that implement the conditions of the abstract reference action in the concrete behaviour. Since this is a complex matter that does not contribute to the objectives of this paper we refrain from discussing it further here.

\section{Context abstraction}

An abstract reference action may represent different alternative concrete activities, which implies that an abstract reference action can be implemented by different alternative activities. These activities can be embedded in a concrete behaviour in different ways. Therefore, we should also be able to determine whether the embedding of the activity in the concrete behaviour conforms to the embedding of the abstract reference action in the abstract behaviour.

In our approach we use a method to deduce the abstract behaviour from a concrete behaviour that is obtained through action refinement. This method starts with the definition of an abstract reference action for each activity and for each action that has not been refined. Once the abstract reference actions have been defined, we apply abstraction rules which allows one to abstract from the remaining actions of the activities. These remaining actions are called inserted actions, since they are considered as being 
'inserted' in the abstract behaviour. Abstraction rules are recursively applied until a behaviour which consists exclusively of abstract reference actions is obtained. This behaviour is the abstraction of the concrete behaviour.

The following steps are identified for the above method:

1. for each activity, identify the condition, in terms of the occurrences of final actions, that corresponds to the occurrence of the abstract reference action. Considering the three cases of activity ending in section 4 , this condition is the occurrence of (i) the final action, (ii) all final actions, and (iii) one of the final actions, respectively;

2. for each activity, integrate the causality relations of the final actions to define the causality relation of the $a b-$ stract reference action;

3. replace the conditions defined in step 1 by their corresponding abstract reference actions in the causality relations of other actions;

4. abstract from non-final actions of activities using the $a b$ straction rules for context abstraction discussed below;

5. proceed with step 3 until all non-final actions of activities, i.e. the remaining inserted actions, are removed.

The remainder of this section discusses some general rules for context abstraction.

\subsection{Behaviours without exclusion}

Initially we consider a concrete behaviour which is defined in terms of causality, involves no exclusion and no action attributes. Since circular definitions in causality relations are not allowed, an action will never be found in its conditions nor in the condition of its enabling actions. Furthermore, since causality is transitive the following abstraction rule can be defined:

\section{Abstraction Rule 1:}

an inserted action that is an enabling action of an action of the concrete behaviour can be replaced by the condition of the inserted action as defined in its causality relation.

Figure 5 depicts an example of the application of abstraction rule 1. Action $e$ is an inserted action in the behaviour $B:=\{$ start $\rightarrow b$, start $\rightarrow c$, start $\rightarrow d, b \wedge c \rightarrow e, d \vee e \rightarrow a\}$, which is a sufficient condition for the occurrence of $a$. When we abstract from $e$ we obtain a more abstract behaviour in which the occurrences of both $b^{\prime}$ and $c^{\prime}$ are a sufficient condition for the occurrence of $a^{\prime}$.

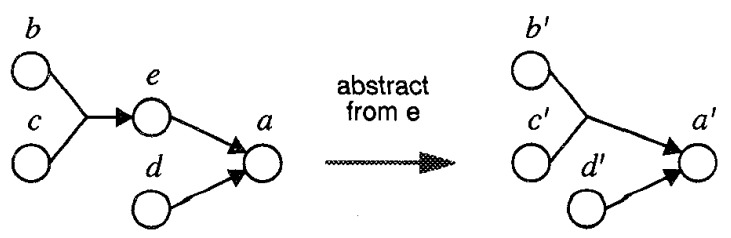

Figure 5: Application of abstraction rule 1
Analogously, if action $e$ would be a necessary condition for $a$ in the concrete behaviour, then the occurrences of both $b^{\prime}$ and $c^{\prime}$ would be a necessary condition for the occurrence of $a^{\prime}$ in the abstract behaviour.

Action attributes. Abstraction rule 1 does not consider abstraction from references to attribute values of inserted actions. Since references to attribute values are only possible with enabling actions, rules to abstract from attribute values of inserted actions are applied in combination with abstraction rule 1 . The abstraction rules for information value and time attributes are defined below.

Abstraction Rule la:

references to information values of inserted actions can be substituted by their values or constraints.

Abstraction Rule $1 b$ :

references to time attribute values of inserted actions can be substituted by their values or constraints.

\subsection{Behaviours containing exclusion}

Unlike causality, exclusion is not transitive. This implies that abstraction rule 1 can not be applied to any arbitrary behaviour containing exclusion. In the sequel we identify behaviours to which abstraction rule 1 can be applied, and develop new rules for those situations to which this rule can not be applied.

No exclusion relations involving inserted actions. Since only inserted actions are removed from a concrete behaviour, abstraction rule 1 can still be applied in case inserted actions are not involved in any exclusion relation. This implies the following more general condition for the application of abstraction rule 1:

- the condition of an inserted action does not contain the non-occurrence of any action of the concrete behaviour, and

- the non-occurrence of inserted actions is not used in the condition of any action.

Figure 6 depicts two behaviours involving exclusion in which abstraction rule 1 can be applied.

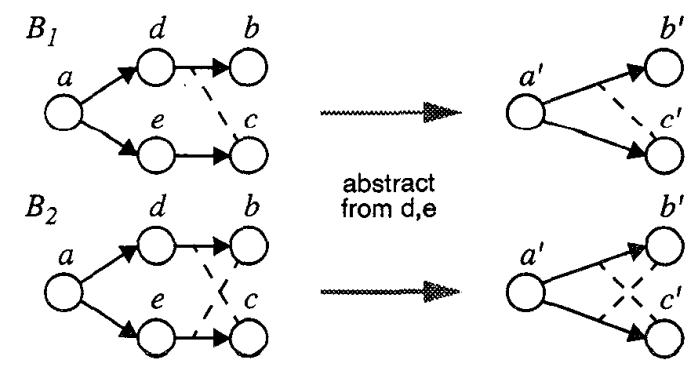

Figure 6: Exclusion not involving inserted actions 
Making uncertainty explicit. The abstract behaviour may define that an abstract reference action may or may not occur using the probability attribute. The conditions for this uncertainty with respect to the occurrence of an abstract reference action may be defined explicitly in the concrete behaviour in terms of the occurrences and non-occurrences of inserted actions. In order to allow abstraction of such concrete behaviours, additional abstraction rules have to be defined to handle the cases in which abstraction rule 1 can not be applied as described above. Two abstraction rules to handle some of these cases are discussed below.

Removal of exclusion. Figure 7 depicts two behaviours $B_{1}$ and $B_{2}$ in which abstraction rule 1 can not be applied to abstract from an inserted action $d$ which is disabled by action $c$ and action $e$, respectively. When abstracting from $d$ it is not possible to explicitly represent the specific situations in which $b^{\prime}$ occurs or does not occur in the abstract behaviour. Replacing action $d$ by its condition $a \wedge \neg c$ in $B_{1}$ and $a \wedge \neg e$ in $B_{2}$ would result in an incorrect abstraction, since the concrete behaviours allow $b$ to occur after $c$ or $e$ has occurred, respectively.

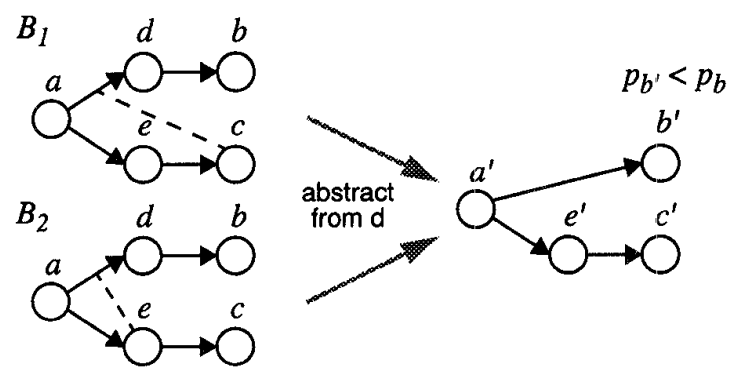

Figure 7: Removal of exclusion

Instead, the exclusion relation is removed and the probability attribute of $b^{\prime}$ is used to represent that this action may or may not occur. In general the probability attribute value of $p_{b^{\prime}}$ is smaller than the probability attribute value of $p_{b}$, since the probability that $b$ occurs after that $d$ has occurred is bigger than the probability that $b$ occurs after that $a$ occurs, due to the possibility that $d$ does not occur after $a$. Therefore the possibility that $b^{\prime}$ does not occur after $a^{\prime}$ due to the inserted actions should be explicitly represented in the probability attribute of $b^{\prime}$.

The following rule is defined to abstract from an inserted action for which the non-occurrence of another action of the concrete behaviour is a necessary condition:

Abstraction Rule 2:

consider an inserted action that is a necessary condition for an action, say $a$. A necessary exclusion condition of this inserted action can be discarded when the inserted action is replaced by its conditions. The probability attribute value of the abstraction of action $a$ in the abstract behaviour decreases with respect to the probability attribute value of $a$.

Inversion of causality. Figure 8 depicts two behaviours $B_{1}$ and $B_{2}$ in which abstraction rules 1 and 2 can not be applied to abstract from an inserted action $e$ which may disable the occurrences of action $b$ and action $d$, respectively. Neither can action $e$ be replaced by its condition nor can the exclusion relation be removed in the abstract behaviour.

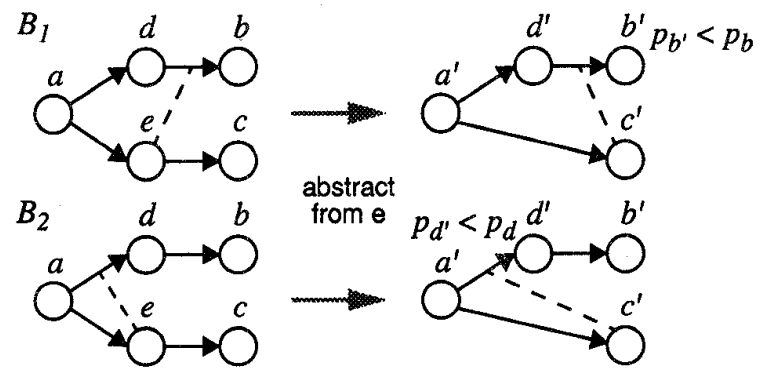

Figure 8: Inversion of causality

In order to represent that action $b^{\prime}$ or action $d^{\prime}$ can be disabled, respectively, the causality relation of action $c$ is inverted. This is based on the observation that in case $e$ does not occur, $c$ does not occur either, which means that the nonoccurrence of $e$ implies the non-occurrence of $c$. Therefore, the non-occurrence of $e$ can be replaced by the non-occurrence of $c$. Furthermore, the probability attribute values of $p_{b^{\prime}}$ in $B_{1}$ and $p_{d^{\prime}}$ in $B_{2}$ are smaller than the probability attribute value of $p_{b}$ and $p_{d}$, respectively, since the probability that action $b$ or action $d$ is disabled by $e$ is bigger than the probability that action $b^{\prime}$ or action $d^{\prime}$ is disabled by $c^{\prime}$, respectively.

The following rule is defined to abstract from an inserted action of which the non-occurrence is a necessary condition for another action:

Abstraction Rule 3:

an inserted action that is a disabling action of an action, say $a$, can be replaced by another disabling action, say $b$, where $b$ is enabled by the inserted action. The probability attribute value of the abstraction of action $a$ in the abstract behaviour decreases with respect to the probability attribute value of $a$. This abstraction is only possible if we can abstract from possible references in $b$ to attribute values of the inserted action.

\subsection{Action choice}

Abstraction rules 2 and 3 apply to singular exclusion relations between two actions; i.e. the occurrence of one action excludes the other, or vice versa, but not both.

In case of a choice between two actions, i.e. the occurrence of one action excludes the other, and vice versa, appli- 
cation of abstraction rules 2 and 3 gives incorrect abstractions. The following abstraction rule is defined to abstract from inserted actions in case of action choice:

\section{Abstraction Rule 4:}

consider that the occurrence of an inserted action is a necessary condition of an action, say $a$. A choice between this inserted action and another action, say $b$, is inherited by actions $a$ and $b$.

Figure 9 depicts two concrete behaviours containing a choice between two inserted actions and a choice between an inserted and a final action, respectively, and their abstractions obtained by applying abstraction rule 4 .

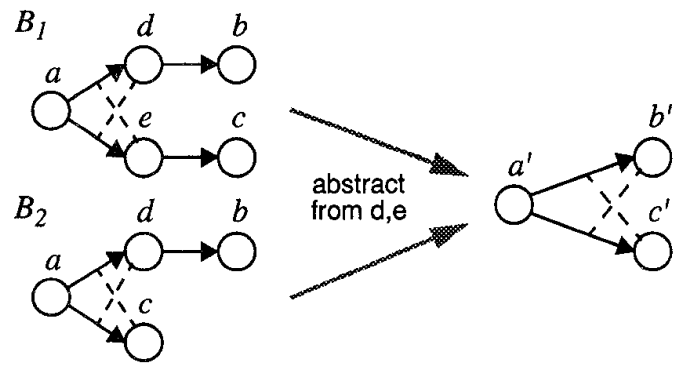

Figure 9: Inheritance of choice

Limitations. Abstraction rules 1 to 4 handle most cases of behaviour that can be found in practise, however, these abstraction rules do not handle exclusion as a sufficient condition. The definition of abstraction rules for these cases is left for further study.

\section{Example}

Figure 10 depicts an example of a concrete behaviour which represents a connection-oriented data transfer service with the following characteristics:

- there is a calling user, at access point $a$, which initiates connection establishment;

- there is a called user, at access point $b$, which accepts or rejects the requested connection;

- only the calling user is allowed to send (four) data unis;

- only the calling user is allowed to initiate a disconnect.

Figure 10 refrains from representing attribute values, for the sake of conciseness.

The concrete behaviour consists of three types of activities: a connect activity, four data activities, and a disconnect activity. These activities are a refinement of their corresponding abstract reference actions in the abstract behaviour depicted in figure 10 .

The connect activity contains a disjunction of two final actions: concnf, which represents the successful establishment of a connection, and conrej, which represents the rejection of the requested connection. The occurrence of abstract reference action conn corresponds to the occurrence of either one of these two actions. The information value attribute of action conn can be used to model both possible outcomes of the connect activity, which could be represented by two values "confirm" and "reject", respectively. In case the value "reject" is established the behaviour stops.

According to abstraction rule 4 the choice between inserted actions conrsp (connection response) and conref (connection refusal), is inherited by the final actions concnf

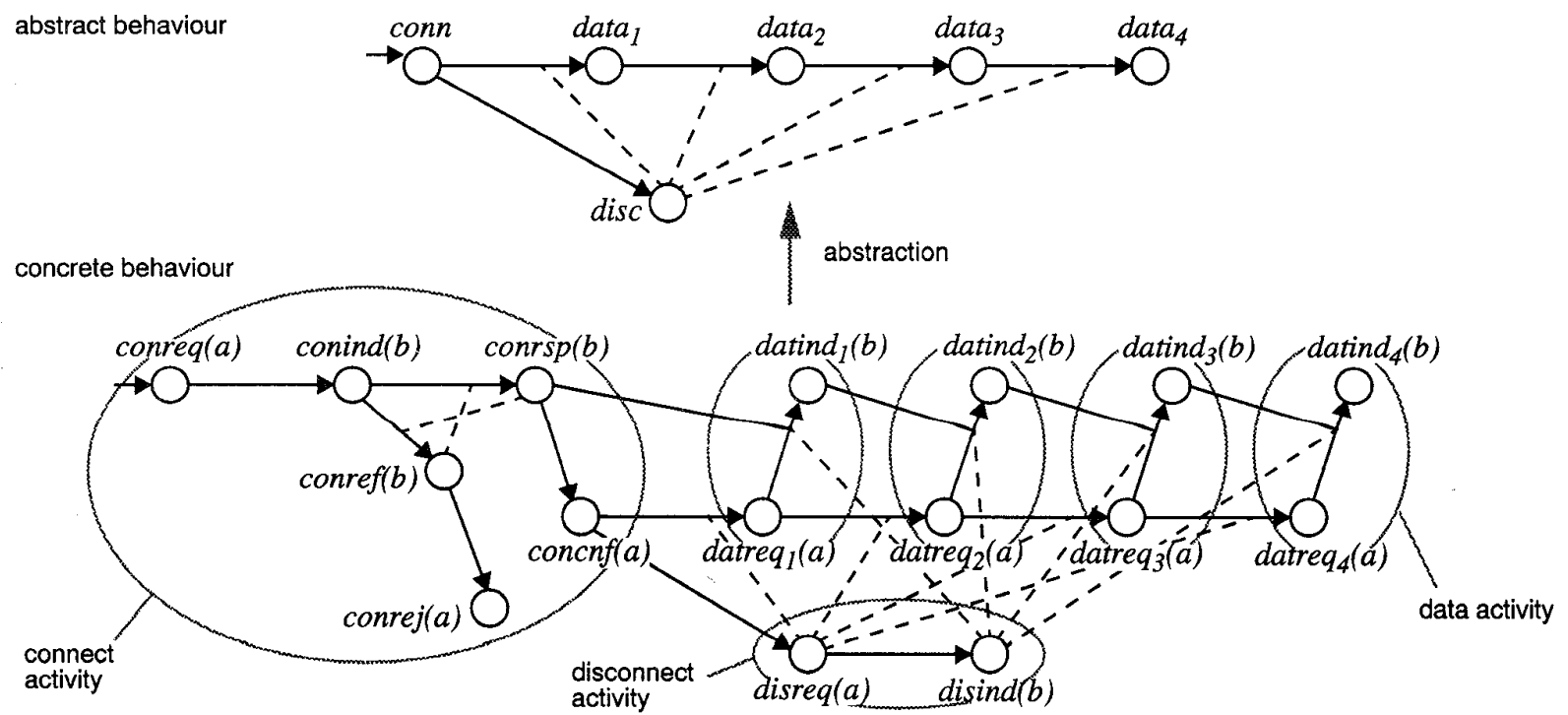

Figure 10: Connection-oriented behaviour 
and conrej. Abstraction rule 1 is used to abstract from inserted actions conind (connection indication) and conreq (connection request), respectively.

The disconnect activity consists of a single final action disind (disconnect indication) and an inserted action disreq (disconnect request). The occurrence of disind corresponds to the occurrence of abstract reference action disc, representing the termination of the connection. Abstraction rule 3 is used to abstract from disreq.

Similarly, the data activities consist of a single final action datind $_{x}$ (data indication) and an inserted action datreq (data request). The occurrence of datind $x_{x}$ corresponds to the occurrence of abstract reference action data $_{x}$, representing the exchange of a data unit. Inserted action datre $_{x}$ is removed using abstraction rule 2 .

The abstract behaviour models direct communication between the calling and called user, abstracting from the geographical distribution between both users. All abstract reference actions occur at a single abstract interface, which is an abstraction of access points $a$ and $b$. This implies that the action refinement design operation has been used to distribute the establishment of information values over two remote locations $a$ and $b$, which are interconnected by some connection-oriented data transfer service provider.

In most connection-oriented services, connection refusal and connection reject are performed using disconnect request and disconnect indication primitives. Application of the rules for action refinement generates a precise argument in favour of modelling the actions connection refusal/reject and disconnect request/indication as distinct actions, since these actions belong to different activities.

\section{Conclusions and discussion}

A design model can only effectively support the design process if the relation between designs at different abstraction levels is also considered. Many design models limit this relation to comparing the observable behaviour of different designs at different abstraction levels. However, comparing the observable behaviour of two behaviours implies that each observable action in one behaviour has one corresponding observable action in the other behaviour, with the same attribute values.

In order to support the implementation of abstract actions by (complex) activities it is also necessary to consider the relation between two behaviours at different abstraction levels, in which an action in the abstract behaviour is replaced by an activity in the concrete behaviour. The action refinement design operation as discussed in this paper deals with this relation.

This paper presents a conceptual basis to perform action refinement in a general way, which is based on a careful consideration of the 'action' and 'causality relation' archi- tectural concepts. Abstraction rules are defined to determine whether an activity is a valid refinement of an abstract action, considering the contexts in which they are embedded. These abstraction rules can be applied to many different behaviour patterns, but more rules have to be defined to handle all possible behaviour patterns, in particular those involving causality relations which contain sufficient causality conditions. We believe this approach enables a more general definition of action refinement than the ones normally found in the literature (e.g. [1] and [6]).

Action refinement is defined to support design steps in a design methodology for distributed systems, which has been discussed amongst others in [2] and [5] and will be extended further in our future work. Part of this work will address the definition of a formal semantics for our design model, which should enable the development of tools to partly automate the action refinement design operation.

\section{Acknowledgements}

This work is partly funded by the Dutch Ministry of Economic Affairs in the Platinum project.

\section{References}

[1] L. Aceto and M. Hennessy. Adding action refinement to a finite process algebra. In J. Leach Albert, B. Monien, and M. Rodríguez Artalejo, editors, Automata, Languages and Programming, volume 510 of Lecture Notes in Computer Science, pages 506-519, Germany, 1991. Springer-Verlag.

[2] L. Ferreira Pires. Architectural notes: a framework for distributed systems development. $\mathrm{PhD}$ thesis, University of Twente, Enschede, the Netherlands, 1994.

[3] L. Ferreira Pires, M. van Sinderen, and C. A. Vissers. Advanced design concepts for distributed systems development. In Proceedings of the Fourth Workshop on Future Trends of Distributed Computing Systems, pages 419-425, Los Alamitos, USA, Sept. 1993. IEEE Computer Society Press.

[4] K. Turner. An architectural semantics for LOTOS. In H. Rudin and C. West, editors, Protocol Specification, Testing and Verification, VII, The Netherlands, 1987. Elsevier Science Publisher B.V. (North-Holland).

[5] C. A. Vissers, M. van Sinderen, and L. Ferreira Pires. What makes industries believe in formal methods. In A. Danthine, G. Leduc, and P. Wolper, editors, Protocol Specification, Testing, and Verification, XIII, pages 3-26, The Netherlands, 1993. Elsevier Science Publishers B.V. (North-Holland).

[6] W. Vogler. Bisimulation and action refinement. Theoretical Computer Science, (114):173-200, 1993. 